\title{
The use of Escherichia coli total antigens as a complementary approach to address seropositivity to Leishmania antigens in canine leishmaniosis
}

\author{
CARLA LIMA ${ }^{1,2,3}$, JOÃO RODRIGO MESQUITA ${ }^{3,4,5}$, HUGO BRANCAL ${ }^{6,7}$, \\ THOMAS VAHLENKAMP ${ }^{8}$, ANA RAFAELA TEIXEIRA ${ }^{1,2}$, LUÍS CARDOSO ${ }^{9}$, \\ CÉLIA AMORIM ${ }^{1,2}$, NUNO SANTARÉM ${ }^{1,2} *$ and ANABELA CORDEIRO DA SILVA ${ }^{1,2,3 *}$ \\ ${ }^{1}$ Instituto de Investigação e Inovação em Saúde, Universidade do Porto, Portugal \\ ${ }^{2}$ Instituto de Biologia Molecular e Celular, Universidade do Porto, Porto, Portugal \\ ${ }^{3}$ Departamento de Ciências Biológicas, Faculdade de Farmácia da Universidade do Porto, Porto, Portugal \\ ${ }^{4}$ Centro de Investigação em Biodiversidade e Recursos Genéticos, Universidade do Porto, Campus Agrário de Vairão, \\ Vairão, Portugal \\ ${ }^{5}$ Escola Superior Agrária, Instituto Politécnico de Viseu, Viseu, Portugal \\ ${ }^{6}$ Clínica Veterinária da Covilhã, Quinta das Ferreiras, Boidobra, Covilhã, Portugal \\ ${ }^{7}$ Faculdade de Ciências da Saúde, Universidade da Beira Interior, Covilhã, Portugal \\ ${ }^{8}$ Faculty of Veterinary Medicine, University of Leipzig, Leipzig, Germany \\ ${ }^{9}$ Departamento de Ciências Veterinárias, Escola de Ciências Agrárias e Veterinárias, Universidade de Trás-os-Montes e \\ Alto Douro (UTAD), Vila Real, Portugal
}

(Received 29 November 2016; revised 24 April 2017; accepted 25 April 2017; first published online 23 May 2017)

SUMMARY

Canine leishmaniosis (CanL) is a major veterinary concern and a public health issue. Serological data are essential for disease management. Several antigens used in serological assays have specificity related problems preventing relevant seropositivity values establishment. Herein we report significant seropositivity level disparity in a study cohort with 384 dogs from eight countries, for antigens traditionally used in CanL - soluble promastigote Leishmania antigens (SPLA) and K39 recombinant protein (rK39): 43.8 and 2.9\% for SPLA and rK39, respectively. To better understand the reasons for this disparity, CanL-associated serological response was characterized using, for complement serological evaluation, a ubiquitous antigen - soluble Escherichia coli antigens (SECAs). Using cohorts of CanL dogs and dogs without clinical evidences of CanL from non-endemic regions of Portugal, the serological response of CanL animals followed specific trend of seropositivity rK39 > SPLA > SECA absent in non-diseased animals. Using receiver operating characteristic curve analysis, these characteristic trends were converted in ratios, SPLA/SECA, rK39/SECA and rK39/ SPLA, that presented high predictive for discriminating the CanL cohort that was potentiated when applied in a scoring system involving positivity to four out of five predictors (rK39, SPLA, SPLA/SECA, rK39/SECA and rK39/ SPLA). In fact, this approach discriminated CanL with similar sensitivity/specificity as reference antigens, diminishing seropositivity in European cohort to $1 \cdot 8 \%$. Ultimately, non-related antigens like SECA and seropositivity ratios between antigens enable different perspectives into serological data focusing on the search of characteristic serological signatures and not simple absolute serology values contributing to comprehensive serological status characterization.

Key words: Canine leishmaniosis, rK39, serosurveillance, Leishmania, ELISA, seropositivity.

\section{INTRODUCTION}

Canine leishmaniosis (CanL), caused by Leishmania infantum (syn. Leishmania chagasi), is endemic in the Mediterranean basin, South America and Southern Asia, being a major veterinary concern (Saridomichelakis, 2009; Ready, 2010; Noli and Saridomichelakis, 2014; Wylie et al. 2014). These protozoa may also induce human visceral leishmaniasis, making CanL not just a veterinary problem but also a public health issue due to the zoonotic potential of the infection (Michalsky et al. 2007; Sousa

* Corresponding author: Instituto de Investigação e Inovação em Saúde, Universidade do Porto, Portugal. E-mail: cordeiro@ff.up.pt or cordeiro@ibmc.up.pt and santarem@ibmc.up.pt et al. 2011; Noli and Saridomichelakis, 2014). CanL control is based on vaccination, transmission prevention by the insect vectors and also diseased animals treatment (Palatnik-de-Sousa, 2012; Courtenay et al. 2014; Silva et al. 2014). The CanL diagnosis is based on clinical signs and demonstration of infection by direct visualization of parasites and/or genetic material in conjunction with quantitative serology (Michalsky et al. 2007; Paltrinieri et al. 2010; Solano-Gallego et al. 2011). Of paramount importance for disease management is the detection of dogs that have no leishmaniosis specific signs but are infected. It is well established that infected dogs without clinical signs are capable of perpetuating the zoonotic life cycle of the parasite (Molina et al. 1994; Moreno and Alvar, 2002). Therefore,

Parasitology (2017), 144, 1384-1393. C Cambridge University Press 2017. This is an Open Access article, distributed under the terms of the Creative Commons Attribution licence (http://creativecommons.org/licenses/by/4.0/), which permits unrestricted re-use, distribution, and reproduction in any medium, provided the original work is properly cited. 
low-accuracy serological assays can have significant impact in the global strategy to combat disease progression originating seropositivity levels that are not translatable into meaningful epidemiological data influencing policies associated with specific control measures. In fact, cumulative evidence point to a low specificity ( $\mathrm{Sp}$ ) of several serological assays that hamper the detection of Leishmania infection in dogs (Gomes et al. 2008; Morales-Yuste et al. 2012; Arruda et al. 2016; Elshafie et al. 2016; Fraga et al. 2016). Moreover, detection of L. infantum vectors across central European countries like Switzerland, Germany and Hungary (Naucke et al. 2008; Ready, 2010), in conjunction with global warming, might open new areas to endemicity, through the adaptation of the vectors to new latitudes (Geisweid et al. 2013; Millan et al. 2014). In fact, disease is advancing northwards, with reports of infected animals in traditionally non-endemic areas (Saari et al. 2000; Dujardin et al. 2008; Ready, 2010; Miro et al. 2012; Carvalho et al. 2015; Maia and Cardoso, 2015). Therefore, more than ever, it is essential to have adequate serosurveillance systems and tools to monitor and control infection progression into new regions.

Herein we evaluate serological response of an uncharacterized cohort of animals from distinct geographical backgrounds to two known Leishmania antigens [soluble promastigote Leishmania antigens (SPLA) and K39 recombinant protein (rK39)] to determine the adequacy of these antigens for serological surveys in non-endemic regions. To exclude any inter-laboratory variability, we used CanL-positive and negative cohorts of dogs to determine the cut-offs that are able to distinguish diseased from non-diseased animals for both antigens. The generated cut-offs were applied to the test cohort and levels of seropositivity compared. The unrelated soluble Escherichia coli antigen (SECA) was also used to address the $\mathrm{Sp}$ of the serological response to the Leishmania antigens. Finally, the ratios between the cut-off normalized responses to the antigens were evaluated for their capacity to predict disease in the control cohorts and then used as part of a scoring system that addresses seropositivity with clinical value.

\section{MATERIALS AND METHODS}

\section{Canine sera}

Group CanL+ $(n=29)$ : sera from dogs living in geographical regions of Portugal where CanL is endemic, which were brought to a veterinary hospital, clinic or anti-rabies campaign and that presented at least two clinical signs compatible with the disease (viz. lymphadenomegaly, alopecia, dermatitis, skin ulceration, keratoconjunctivitis, onychogryphosis, lameness, epistaxis, anorexia and weight loss). These animals were also seropositive for anti-Leishmania antibodies by the direct agglutination test (DAT) (cut-off titre $=400$ ) and/or positive for the presence of amastigotes in bone marrow or lymph node aspirates.

Group CanL- $(n=121)$ : sera from dogs that visited a veterinary clinic in a Portuguese region considered to be non-endemic for CanL, Serra da Estrela mountain in central Portugal. All were seronegative by DAT (titre $<100)$.

Group Europe $(n=384)$ : sera from dogs without clinical information, with unknown serological status, that visited veterinary clinics in several countries of Europe, namely Denmark $(n=50)$, France $(n=50)$, Germany $(n=47)$, Hungary $(n=47)$, the Netherlands $(n=42)$, Poland $(n=50)$, Portugal $(n=50)$ and the $\mathrm{UK}(n=48)$.

\section{Antigens}

Three different antigens were used for enzymelinked immunosorbent assay (ELISA): SPLA, SECA and Leishmania rK39. For SPLA, L. infantum promastigotes were obtained as previously described (Santarem et al. 2010). Parasites were washed three times with phosphate-buffered saline (PBS), $\mathrm{pH} 7 \cdot 4$ and centrifuged at $3500 \mathrm{~g}$ for 10 min at $4{ }^{\circ} \mathrm{C}$. The pellet was resuspended in PBS containing $1 \mathrm{~mm}$ phenylmethylsulfonyl fluoride (PMSF) protease inhibitor and submitted to 10 freeze-thaw cycles for rupture of the parasites. This suspension was centrifuged at $13000 \boldsymbol{g}$ for 30 min at $4{ }^{\circ} \mathrm{C}$ and the supernatant was recovered, quantified by DC (detergent compatible) ${ }^{\mathrm{TM}}$ Protein Assay (BioRad, Munich, Germany), and stored at $-80^{\circ} \mathrm{C}$ in single aliquots.

The rK39 antigen obtained from Dr Steven Reed, from Infectious Disease Research Institute (Seattle, Washington, USA) was suspended in mili-Q water, quantified and stored at $-80^{\circ} \mathrm{C}$ in single-use aliquots.

For SECA, E. coli DH5- $\alpha$ strain was plated in an agar plate and incubated at $37^{\circ} \mathrm{C}$ overnight $(\mathrm{O} / \mathrm{N})$. Then, a colony was transferred to $10 \mathrm{~mL}$ of Luria Bertani broth (Sigma-Aldrich, St. Louis, USA) and incubated at $37^{\circ} \mathrm{C} \mathrm{O} / \mathrm{N}$. The next day, the optical density (OD) of the culture was measured at $600 \mathrm{~nm}$ (initial OD should be nearly $0 \cdot 1$ ) and left to grow for $3 \mathrm{~h}$. The culture was then centrifuged at $4000 \boldsymbol{g}$ for $5 \mathrm{~min}$. The pellet was resuspended in $1 \mathrm{~mL}$ of PBS containing $1 \mathrm{mM}$ of PMSF and submitted to 10 freeze-thaw cycles for rupture of the bacteria. The suspension was then centrifuged at $13000 \boldsymbol{g}$ for $30 \mathrm{~min}$ at $4{ }^{\circ} \mathrm{C}$ and the supernatant was recovered, quantified by DC (detergent compatible) ${ }^{\mathrm{TM}}$ Protein Assay (BioRad, Munich, Germany) and stored at $-80^{\circ} \mathrm{C}$ in single-use aliquots.

\section{ELISA}

Ninety-six-well flat-bottom microtitre plates (Greiner Bio-One, Frickenhausen, Germany) were 


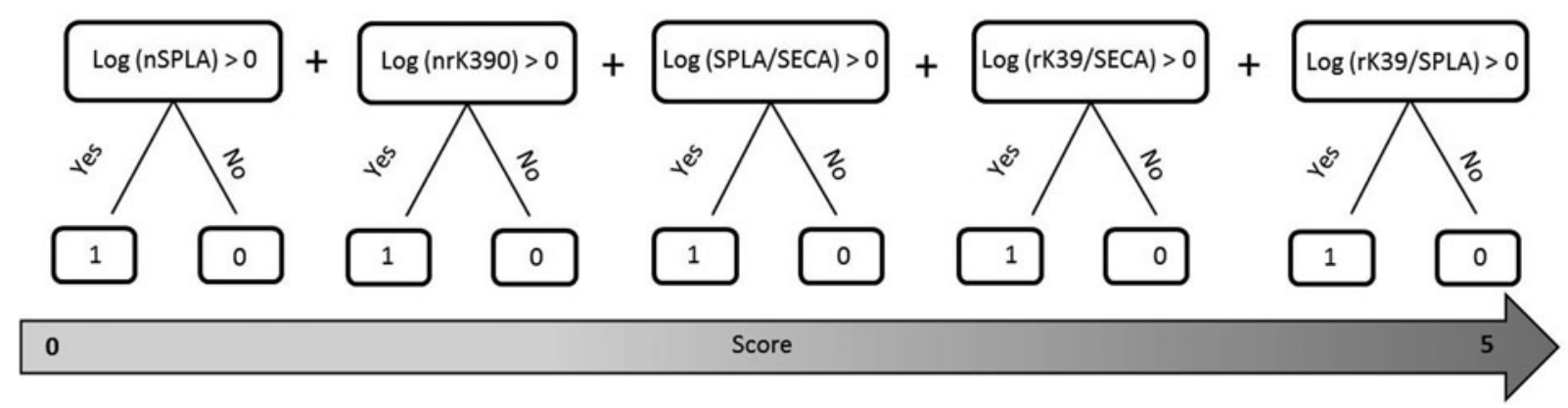

Fig. 1. Evaluation of seropositivity of each individual serum evaluated as a cumulative score. Each individual serum was 'scored' as $n$ SPLA $+n$ rK39 $+n$ SECA + SPLA/SECA + rK39/SECA + rK39/SPLA using the following premise: each serology value above or equal to the cut-off values of $n$ SPLA, $n \mathrm{rK} 39, n$ SECA, SPLA/SECA, rK39/SECA and rK39/ SPLA was transformed into a score of ' 1 ' originating a cumulative score ranging from 0 to 5 to each individual sample.

coated with $50 \mu \mathrm{L}$ of $0 \cdot 1 \mathrm{M}$ carbonate buffer, $\mathrm{pH}=$ $9 \cdot 6$, with $10 \mu \mathrm{g} \mathrm{mL}{ }^{-1}$ of either SPLA or SECA, or $1 \mu \mathrm{g} \mathrm{mL} \mathrm{m}^{-1}$ of $\mathrm{rK} 39$. Plates were incubated $\mathrm{O} / \mathrm{N}$ at 4 ${ }^{\circ} \mathrm{C}$ and blocked with $200 \mu \mathrm{L}$ of PBS low-fat milk (3\%) at $37^{\circ} \mathrm{C}$ for $1 \mathrm{~h}$. Next, plates were washed with PBS-Tween 0.05\% (PBS-T), and the sera, positive and negative controls, diluted at $1: 1500$ in PBS-T low-fat milk (1\%), were dispensed in triplicate $(100$ $\mu \mathrm{L}$ per well) and incubated at $37^{\circ} \mathrm{C}$ for $30 \mathrm{~min}$. After a washing step, $100 \mu \mathrm{L}$ per well of conjugate secondary anti-dog IgG antibody conjugated with horseradish peroxidase (Sigma, St. Louis, USA) diluted at $1: 1176 \cdot 5$, was added and the plates were incubated at $37^{\circ} \mathrm{C}$ for $30 \mathrm{~min}$. Plates were washed and incubated with $0.5 \mathrm{mg} \mathrm{mL}^{-1}$ of $o$-phenylenediamine dihydrochloride (Sigma, St. Louis, USA) for $10 \mathrm{~min}$ in dark. Reaction was stopped with $50 \mu \mathrm{L}$ per well of $\mathrm{HCl}(3 \mathrm{M})$. Absorbance was read at 492 $\mathrm{nm}$ in an automatic reader (Synergy 2, BioTek Instruments, Winooski, USA).

All samples and antigens were assayed in at least two independent assays.

\section{Statistical analysis}

Receiver operating characteristic (ROC) curves were generated using sera from groups CanL+ and CanL-, using GraphPad Prism version 5.00 for Windows, GraphPad Software, San Diego California USA, www.graphpad.com. A 95\% confidence interval for the area under the ROC curve was considered.

Cut-off values were inferred through these curves for each antigen [by choosing the best compromise between sensitivity ( $\mathrm{Se}$ ) and $\mathrm{Sp}$ associated with the ROC curve], and values of $\mathrm{Se}, \mathrm{Sp}$, false negatives $(\mathrm{FN})$, false positives (FP), positive predictive value and negative predictive value were calculated for the samples for each group (Parikh et al. 2008). Optical densities of each sample were normalized by division with the corresponding cut-off value ( $n$ SPLA, $n$ rK 39 and $n$ SECA) and the logarithm of this ratio was applied for graphical representation $[\log (n \mathrm{SPLA}), \log (n \mathrm{rK} 39)$ and $\log (n \mathrm{SECA})]$. The cut-off normalized values $(n)$ were used to assess the ratio between the antigens: SPLA/SECA (ratio between $n$ SPLA and $n$ SECA), rK39/SECA (ratio between $n \mathrm{rK} 39$ and $n \mathrm{SECA}$ ) and rK39/SPLA (ratio between $n \mathrm{rK} 39$ and $n \mathrm{SPLA}$ ). A score evaluation method was established based on the cut-off values inferred from the ROC curves for the ratios and the single antigens. A binary score ( 1 or 0$)$ was applied to represent seropositivity (arbitrary value of 1) or seronegativity (arbitrary value of 0 ) to the five serological parameters previously established and whose cut-off values was determined by ROC curve analysis: rK39, SPLA, rK39/SECA, SPLA/ SECA and rK39/SPLA. Total cumulative score for each individual sample ranged from ' 0 ' to ' 5 ' (Fig. 1).

\section{RESULTS}

ROC curve analysis was performed to define cut-off values for rK39 and SPLA using sera from dogs of CanL+ and CanL- groups (Supplementary Fig. S1A, S1B and Table S1). Cut-off values for rK39 and SPLA were $0 \cdot 127$ and $0 \cdot 074$, respectively. These cut-offs enabled a Se of 100\% (29/29) for both antigens with $\mathrm{Sp}$ of $100 \%$ in both cases $(0 / 121)$ (Supplementary Table S1). When the cut-offs were applied to the European cohort, they originated a global seropositivity of $43 \cdot 8 \%(168 / 384)$ and $2 \cdot 9 \%$ $(11 / 384)$ for the individual antigens, SPLA and rK39, respectively (Table 1, Fig. 2A-C). If seropositivity to both antigens was used as a requisite, then $2 \cdot 3 \%(9 / 384)$ of the cohort was seropositive (Table 1).

Observing the generated seropositivity data disparity for antigens that excelled at discriminating CanL from non-CanL dogs, two possibilities were considered: either the SPLA was detecting FP or rK39 was detecting FN. Considering that both antigens presented very high $\mathrm{Sp}$ and $\mathrm{Se}$, the cut-off stringency was increased to decrease the number of FP (Supplementary Table S2 and Fig. 3A). For seropositivity associated with both antigens to have 
Table 1. Seropositivity levels in the different study cohorts for SPLA and rK39 antigens

\begin{tabular}{lllll}
\hline \hline & SPLA $(\%)$ & rK39 $(\%)$ & SPLA $\Lambda$ rK39 $(\%)$ & SPLA V rK39 $(\%)$ \\
\hline CanL+ & $100_{(29 / 29)}$ & $100_{(29 / 29)}$ & $100_{(29 / 29)}$ & $100_{(29 / 29)}$ \\
CanL- & $0_{(0 / 121)}$ & $0_{(0 / 121)}$ & $0_{(0 / 121)}$ & $0_{(0 / 121)}$ \\
Europe & $43 \cdot 8_{(168 / 384)}$ & $2 \cdot 9_{(11 / 384)}$ & $2 \cdot 3_{(9 / 384)}$ & $44 \cdot 3_{(170 / 384)}$ \\
\hline \hline
\end{tabular}

a Positive for both antigens simultaneously.

b Positive for at least one of the antigens.

epidemiological relevance, then the increase in cutoff stringency should enable a decrease of FP associated with SPLA leading seropositivity levels similar to rK39. In the European cohort, using two cut-offs (cut-off value $\times 2$ ) for $r K 39$, the number of seropositive animals is reduced to just one, PT30 (Fig. 3B). For SPLA, 66/384 (17.2\%) animals remained seropositive when two cut-offs were used (Supplementary Table S2). Comparing these SPLA-seropositive animals with the original 11 animals seropositive for $\mathrm{rK} 39$, only six animals were seropositive to $\mathrm{rK} 39$ and SPLA using two SPLA cut-offs (Fig. 3B). From 3 to 7 SPLA cutoffs, only three animals were seropositive for both antigens - when compared once again to the original rK39-positive animals (Fig. 3B). The sample with highest seropositivity to both antigens was PT30 (Fig. 3B).

The geographic distribution of seropositive animals was not random, ranged from 0 to $86 \%$. Poland presented (43/50) seropositive animals, while France presented $0 / 50$ animals to SPLA (Fig. 4 and Supplementary Table S2). Considering the individual countries, rK39-seropositive animals were mostly from Portugal: 66.3\% (7/11) (Fig. 4A, Supplementary Fig. S2A). Still, three animals were from non-endemic countries [two from Denmark (DK44 and 50) and one from Poland - PL9] and a final one from France (FR2) (Fig. 3). For SPLA, although there was also an over-representation of dogs from Portugal (i.e. $12 \cdot 5 \% ; 21 / 168$ ), this was not as evident as for rK39 (Supplementary Fig. S2). As the groups were proportional, the basal cohort serological response was compared to evaluate if existed a regional influence in the response to the antigens (Supplementary Tables S3 and S4). For rK39, the dogs from Germany had a basal median response significantly different from Hungary and Portugal, while the animals from Portugal were significantly different from all the other cohorts (Supplementary Fig. S2A and Table S3). For SPLA, more countries had significant differences, with the cohort from Poland being the most divergent (Supplementary Fig. S2 and Table S4). As SPLA is an undefined antigen mixture, these variations in seropositivity might correspond to an unspecific response.

To address serological response $\mathrm{Sp}$, an undefined ubiquitous non-related antigen was used, E. coli
DH5- $\alpha$, to evaluate if the serological response pattern was similar to SPLA suggesting a general unspecific response (Fig. 4A and Supplementary Fig. S2B). The SECA antigen had some predictive value to distinguish the control cohorts (Se of $81.5 \%$ and $\mathrm{Sp}$ of $71.9 \%$ ) (Fig. $4 \mathrm{~B}$ and Supplementary Table S1). Notwithstanding, the seropositivity profile against SECA was distinct from the SPLA (Fig. 4A, Supplementary Fig. S2B and Tables S4 and S5). To further address the relationship between these antigens, correlations in the control cohorts and also in each individual country were done (Supplementary Table S6). In the CanL+ cohort, the highest correlation was obtained for rK39 $v$ SPLA $\left(r=0.499, P<0.01 ; r^{2}=0.249\right)$, whereas the lowest was for rK39 vs SECA $(r=$ $\left.-0.010, P=0.952, r^{2}=0.0001\right)$. In the CanLcohort, the highest correlation value was for SPLA vs SECA $\left(r=0.356, P<0 \cdot 001, r^{2}=0 \cdot 127\right)$. At the country level, the most common significant correlation was SPLA vs SECA (3/8), while rK39 correlated positively with SPLA in two countries and with SECA in one country (Supplementary Table S6). The cohort comparison at a country level (absolute values and correlation data) suggests distinct basal responses to the different antigens influencing the serology absolute values.

To bypass the regional effects on the seropositivity, the ratios between the antigens were analysed for their capacity to predict CanL in the control cohorts. For this, the median normalized singleantigen reactivity in the CanL+ control group was compared (Figs 2 and 4B). The $n \mathrm{rK} 39$ and $n$ SPLA responses were higher than $n \mathrm{SECA}$ in CanL+ cohort. Moreover, this was characteristic of the individual sera from the CanL+ cohort: (27/29) for $n$ SPLA $>n$ SECA and (26/29) $n$ rK39 $>n$ SECA (Supplementary Table S7). Likewise, the response to rK39 was higher than to SPLA in (24/29) of the cohort (Supplementary Table S7). Therefore, these ratios, $\quad[\log (n \mathrm{SPLA} / n \mathrm{SECA})>1 ; \quad \log (n \mathrm{rK} 39 /$ $n \mathrm{SECA})>1 ; \log (n \mathrm{KK} 39 / n \mathrm{SPLA})>1]$ were characteristic of CanL dogs (Supplementary Fig. S3). Consequently, ROC curves analysis was performed for each of the aforementioned individual ratios (Supplementary Fig. S1D-F). The predictive value of these ratios was considerable, although not superior overall to the reference antigens alone (Supplementary Table S1). The Sp of the ratios 


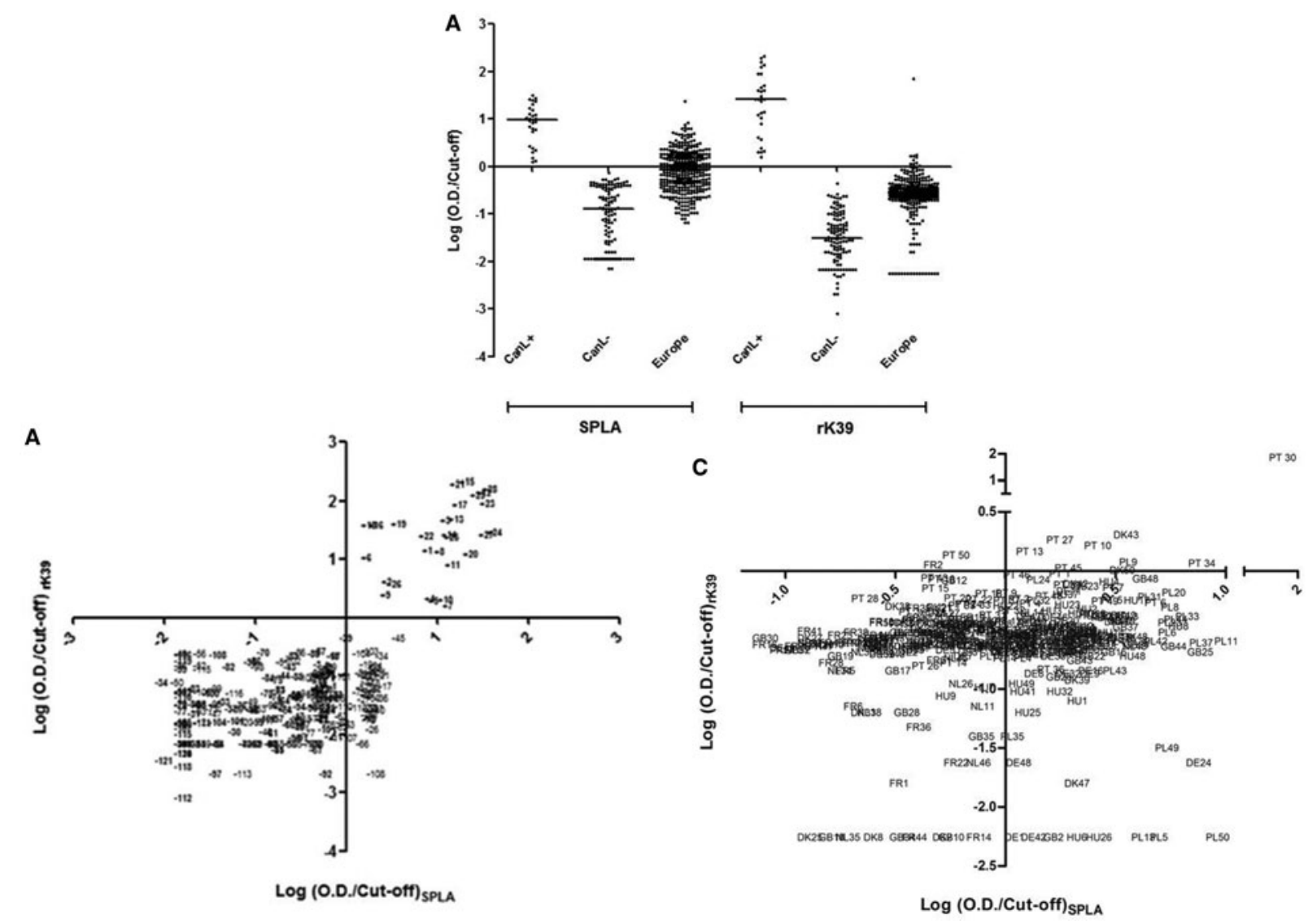

Fig. 2. Reactivity of CanL+, CanL- and European dog sera against SPLA and rK39 antigens (A). Correlation of the seroreactivity against SPLA and rK39 antigens in CanL+ and CanL- cohorts (B) and in European cohort (C). Results are expressed as the logarithm of the optical density (OD) at $492 \mathrm{~nm}$ normalized by the cut-off value for each antigen $(n)$. Bars represent the median for the cohort and each single dot represents the dataset for an individual dog. The data depicted were generated from the average of at least two independent assays done in triplicate.

was $94 \cdot 2,92 \cdot 6$ and $72 \cdot 7 \%$, for $n \mathrm{SPLA} / n \mathrm{SECA}$, $n \mathrm{rK} 39 / n \mathrm{SECA}$ and $n \mathrm{rK} 39 / n$ SPLA, respectively (Supplementary Tables S1 and S8). The lower Se of the assays can be attributed to artefactual responses due to low serological responders that create ratios that are above the cut-off level (see PL38, FR9 and DE47 in Supplementary Table S9). Therefore, the capacity of these ratios to predict disease requires the determination of positivity to reference antigens to exclude the aforementioned artefactual values. Taking this in consideration, a scoring system was created to address not only the positivity to the reference antigens but also to the characteristic ratios described above (Fig. 1). The Cohen's $\kappa$ coefficient was determined for the possible scores to define the best predictive value when compared to both reference antigens. The scores of 4 or 3 presented the best predictive value (Supplementary Table S10). We excluded the score 3 as it enabled positive results that were negative for both reference antigens (Fig. 1). This approach (using a score $\geqslant 4$ ) resulted in an overall Se (96\%) and Sp (100\%) similar to the combined reference antigens (Table 2). When this score system was applied to our cohorts (Table 2), it reduced the number of seropositive animals in the
European cohort to seven (P'T10, P'T30, PL9, PL24, DK43, DK50 and FR2) with an overall seropositivity of $1.8 \% \quad(7 / 384)$ (Table 2 and Supplementary Tables S9-12). This represents a diminution of $36 \%$ in seropositivity when compared with rK39 alone. We also report a diminution of $22 \%$ in seropositivity when compared with rK39 and SPLA combined (Table 1). Only one animal in Europe presented a perfect score of 5 (PT30), the most common score in the CanL+ cohort [82.8\% $(24 / 29)]$, with the remaining six animals presenting a score of 4 . These seven seropositive animals from the European cohort obtained after application of the score methodology were all rK39-seropositive animals with the exception of one sample from Poland (PL24) (Supplementary Fig. S4 and Table S9).

\section{DISCUSSION}

In the present study, the used antigens, SPLA and rK39, are established antigens with reported high sensitivities and specificities and are the base of several reference tests. The cut-off values for two antigens, using a cohort of CanL+ dogs and a 

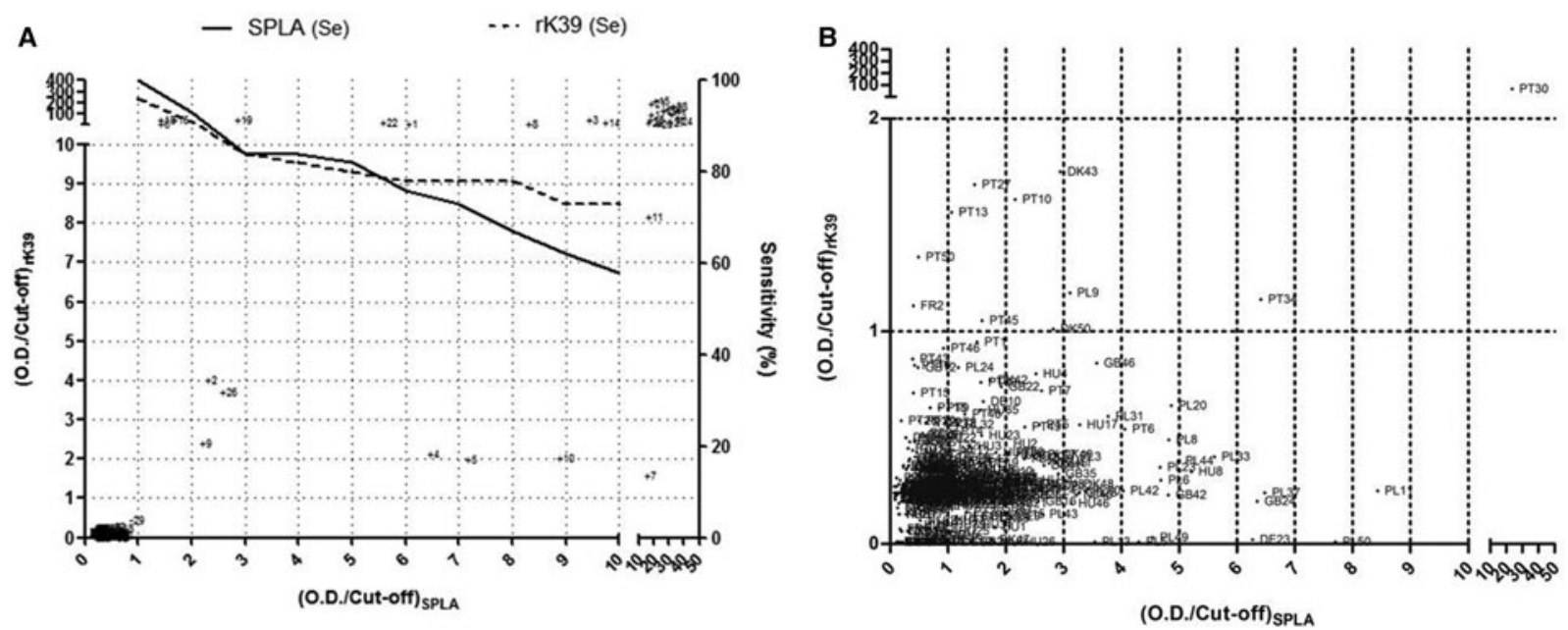

Fig. 3. Representation of seroreactivity against SPLA and rK39 antigens in CanL + and CanL+ cohorts (A), and European cohort (B). Vertical and horizontal dotted lines represent the number of cut-offs ( $n \times$ cut-off) for each antigen. The cut-off-dependent sensitivity is represented in (A) for SPLA and for rK39. Results are expressed as the logarithm of the optical density (OD) at $492 \mathrm{~nm}$ normalized by the cut-off value for each antigen $(n)$. Each single dot represents the dataset for an individual dog. The data depicted were generated from the average of at least two independent assays done in triplicate.
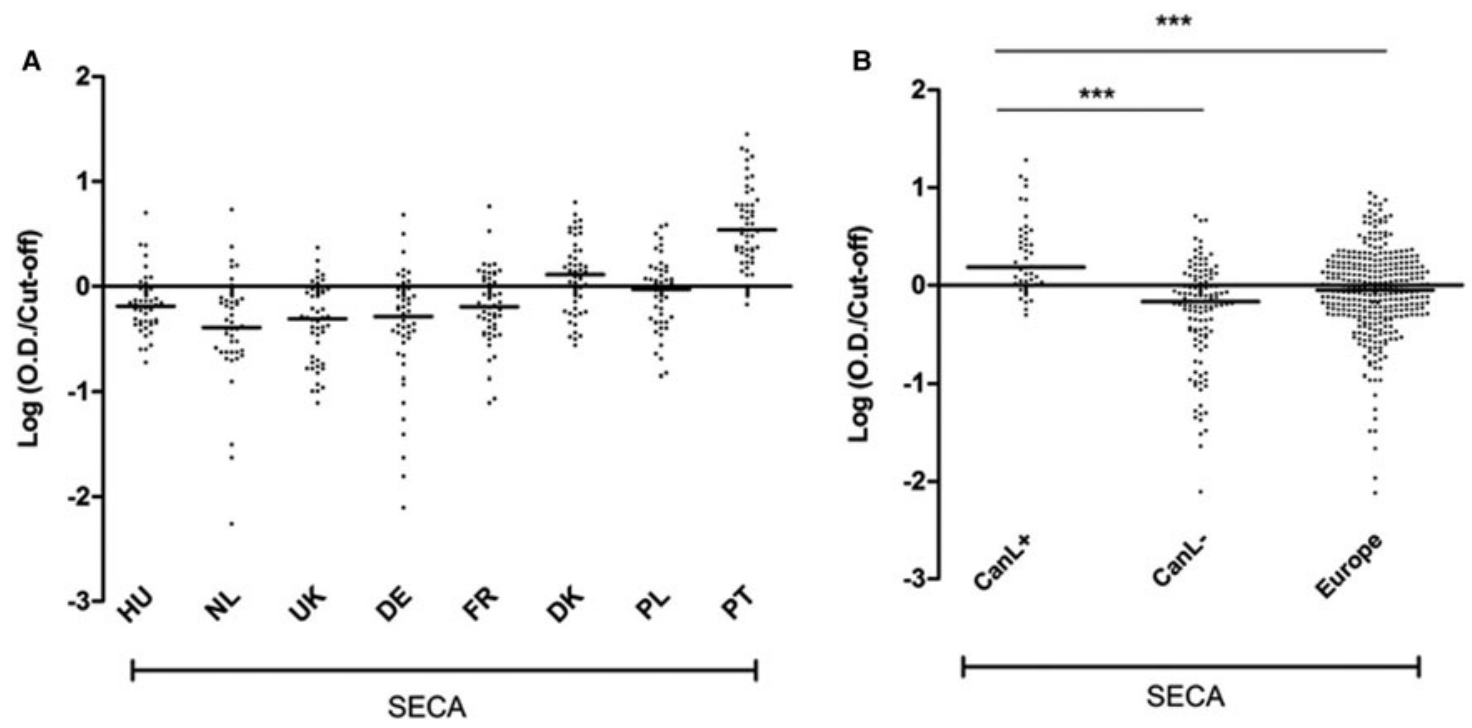

Fig. 4. Reactivity of dog sera against SECA antigen for: (A) individual countries Hungary (HU), The Netherlands (NL), UK, Germany (DE), France (FR), Denmark (DK) and Poland (PL). Results are expressed as the logarithm of the optical density (OD) at $492 \mathrm{~nm}$ normalized by the cut-off value for each antigen $(n)$ and (B) CanL+, CanL- and European cohort. Bars represent the median for the cohort and each single dot represents the dataset for an individual dog. The data depicted were generated from the average of at least two independent assays done in triplicate. Statistical analysis was done by oneway ANOVA and Kruskal-Wallis tests. Statistical significance between groups is 'extremely significant' $(* * * 0 \cdot 0001 \leqslant P<$ $0 \cdot 001)$.

cohort of CanL- dogs, were determined. The obtained cut-offs were similar in absolute value to the ones obtained in previous work from our group (Santarem et al. 2010), in a clear indication that both antigens are capable of consistently discriminating CanL+ from CanL- dogs. When the newly determined cut-offs were applied to a cohort of animals from Europe without clinical characterization, a seropositivity that was distinct for both antigens $(43 \cdot 8 \%$ for SPLA and $2 \cdot 9 \%$ for $\mathrm{rK} 39)$ was obtained. This large difference in seropositivity cannot be attributed to laboratory inter variability, as all assays were performed in the same laboratory, including the ROC curve analysis. The CanL seropositivity levels reported for endemic Mediterranean regions range between 5 and $30 \%$ (Moreno and Alvar, 2002). The overall seropositivity levels obtained for the non-endemic countries was not in accordance with epidemiological data that do not support significant seropositivity levels 
Table 2. Seropositivity levels in the different study cohorts using the scoring system

\begin{tabular}{|c|c|c|c|}
\hline & \multicolumn{3}{|l|}{ Score } \\
\hline & $5^{\prime a \mathrm{a}}(\%)$ & ${ }^{4} 4^{\mathrm{b}}(\%)$ & Total $^{\mathrm{c}}(\%)$ \\
\hline CanL+ & $82 \cdot 8_{(24 / 29)}$ & $13 \cdot 8_{(4 / 29)}$ & $96 \cdot 5_{(28 / 29)}$ \\
\hline CanL- & $0 \cdot 0_{(0 / 121)}$ & $0 \cdot 0_{(0 / 121)}$ & $0 \cdot 0_{(0 / 121)}$ \\
\hline Europe & $0 \cdot 3_{(1 / 384)}$ & $1 \cdot 6_{(6 / 384)}$ & $1 \cdot 8_{(7 / 384)}$ \\
\hline
\end{tabular}

a 5: seropositivity to all serological parameters defined in Fig. 1.

b 4: seropositivity to four out of five serological of the parameters defined in Fig. 1.

c Total: seropositivity to at least four out of the parameters defined in Fig. 1.

in the non-endemic countries from the European cohort (Solano-Gallego et al. 2011). The rK39 seropositivity levels are more in the range of what is reported from the endemic regions, but still nonendemic seropositive samples were identified in Denmark and Poland. These positive animals might be associated with canine population mobility associated with travelling, an issue of growing concern for CanL (Menn et al. 2010; Bart et al. 2013). Leishmania infantum is endemic in southern European countries (Dujardin et al. 2008) that are a highly valued vacation destination generally during the peak of the transmission season. In these conditions, the risk for exposure to the parasite can be as high as $0.23 \%$ for dogs coming from northern Europe (Teske et al. 2002). In fact, the few reported CanL cases in northern Europe are restricted to dogs that have travelled to endemic areas of the Mediterranean basin during periods when phlebotomine sand fly exposure is high, mostly between March and November, or that have to be relocated from those areas (Saari et al. 2000).

SPLA in known to have cross-reactivity with other diseases like babesiosis, Chagas, ehrlichiosis, rickettsiosis and toxoplasmosis, probably due to some epitope conservation generating unspecificity (Gomes et al. 2008; Saridomichelakis, 2009; Silvestre et al. 2009; Zanette et al. 2014; Peixoto et al. 2015). This cross-reactivity was suggested upon correlation of the data from SPLA with SECA (Supplementary Table S6). In the cohorts from three countries, there was a significant correlation between SECA and SPLA, more than between SPLA and rK39. This suggests that, in the absence of a Leishmania-specific serological response, the SPLA might also be recognized by other non-related infectious diseases or immune disorders. This is also suggested by the correlation existing in the CanL- control group for these two antigens. Still, the distinct pattern of basal recognition between SPLA and SECA (Fig. 4A and
Supplementary Fig. S2B) is not suggestive of general unspecific recognition due to immunological disorders, like polyclonal activation. Intriguingly, it will also be important to address if the apparent lack of Sp of SPLA might also be true for other assays that use parasite total antigens, such as IFAT or DAT. The rK39 was less prone to crossreactivity (Fig. 3B). In fact, the general basal serological response was more uniform. This is in agreement with the recognized value of rK39 as a good serological marker for leishmaniosis (Porrozzi et al. 2007). Still, regional differences in antigen performance have been already reported, the performance of $\mathrm{rK} 39$ (for the human visceral leishmaniasis) is known to be diminished in subSaharan Africa (Pattabhi et al. 2010; Bezuneh et al. 2014; Mukhtar et al. 2015). Subtle genetic differences in Leishmania strains are sufficient to influence the performance of the recombinant antigens (Abass et al. 2015).

The adjustment of the cut-off stringency is often used to help in the diagnosis of CanL (Paltrinieri et al. 2010). Therefore, we increased the cut-off stringency (at the cost of decreased Se) (Fig. 3A). Two cut-offs decreased the global seropositivity for SPLA and rK39 to $66 / 384(17 \cdot 2 \%)$ and $1 / 384$ $(0 \cdot 26 \%)$, respectively. Still, a significant difference in readout between both antigens was apparent, as there was no correspondence between SPLA- and rK39-seropositive animals. In fact, the increase in SPLA cut-off stringency also led to a loss of rK39positive animals (standard cut-off), instead of having an enrichment of double positive sera as would be expected for seropositivity related to CanL. Still, it must be referred that existence of dogs with different clinical stage of the disease might also contribute to distinct levels of seropositivity to rK39 and SPLA. It was shown for naturally infected dogs that $\mathrm{rK} 39$, although adequate for detecting symptomatic infections, presented lower capacity to evaluate sub-clinical infections (Santarem et al. 2010). This could justify the failure in enrichment for double positive animals with the cut-off increase. Still, as most countries in the study are non-endemic (exception of Portugal and France), natural exposure to the parasite and asymptomatic infections should not be a decisive factor for the lack of correspondence in seropositivity levels.

To address the possibility of unspecificity of SPLA in the European cohort, SECA was used as a measure of the humoural response that is not specifically directed to Leishmania. Although SECA has some predictive value (probably as a consequence of some level of conservation at the epitope level or due to, in severe cases of the disease, polyclonal activation and effects of co-infections), it presented low Sp (71.9\%). Upon cut-off normalization, it was also evident the clear relationships between the antigens. The median normalized response of the animals of 
the CanL+ control group was highest for rK39 and lowest for SECA (Figs 2A and 4B). This characteristic seropositivity pattern $\mathrm{rK} 39>\mathrm{SPLA}>\mathrm{SECA}$ was also evident for the individual animals (Supplementary Table S8). This was suggestive that the relation between the antigens might have predictive value for the detection of CanL. If fact, the relationship between the three antigens ( $n$ SPLA $/ n$ SECA, $n$ rK39/nSECA, $n$ rK39/nSPLA) presented significant predictive power, as determined be ROC curve analysis (Supplementary Table S1). Still, their direct application in the European cohort did not result in a diminution of seropositivity (Supplementary Table S8). This was not unexpected because the relation is independent on absolute values. Therefore, very low serological results might be seropositive using this approach. Therefore, the unrestricted use of antigen seropositivity ratios present a significant risk of irrelevant artefactual values. Still, these data clearly established that the ratios between the antigens followed a pattern that is in conjunction with positivity to one or both reference antigens was found to be predictive of CanL+ (Fig. 1).

When applied to the European cohort, the score approach originated a global seropositivity of $1 \cdot 8 \%$ (7/384). These seven animals were indistinguishable from the CanL + cohort in at least four evaluated characteristics (one presented a perfect score of 5). This was the animal sorted upon the increase of rK39 threshold (PT30). Only one animal from Poland (PL24), which was classified with a score of 4, was not positive for $\mathrm{rK} 39$ alone. This sample was positive for SPLA and for the three ratios evaluated in the score system ( $n \mathrm{SPLA} / n \mathrm{SECA}, n \mathrm{rK} 39 /$ $n$ SECA and $n \mathrm{rK} 39 / n \mathrm{SPLA})$. Although the absolute value of the OD to rK39 was below the cut-off $(0 \cdot 127)$ for this sample, this difference was only 0.021 (see PL24 in Supplementary Table S9). This is a clear example of problems associated with using absolute values. Subtle differences can place a serum in opposite sides of the threshold. For example, PT45 was seropositive for $\mathrm{rK} 39$, having $0 \cdot 134$ of average OD. Still, in our score system, it was negative with only a score of 3 due to a disproportionally high response to SECA and SPLA (Supplementary Table S9). The high reactivity to SECA prevented positive ratios and consequently preventing the higher score. This very high relative reactivity to SECA was not characteristic of our CanL+ cohort. PT34 is another interesting sample with overall high responses to all tested antigens with average ODs of 0.659 for SECA, 0.447 SPLA and 0.147 for $\mathrm{rK} 39$. Once again, this profile is not characteristic of the infected cohort and might reflect auto-immunity or other complications or a different stage of disease.

In the CanL+ group also, some samples are mentionworthy. The CanL +4 is the sample that prevents a $100 \%$ Se for the score approach. This is the sample with the highest SECA average OD $(2 \cdot 455)$. This might be due to an already advanced stage of the disease resulting in the loss of the characteristic serological pattern of active disease due to other co-infections or even polyclonal activation.

The presented data demonstrate that the evaluation of seropositivity is a complex issue that is often oversimplified. We report that complex mixtures of antigens like SPLA are prone to crossreactivity making them more unreliable for serosurveillance studies than rK39. Recombinant antigens like rK39 were less afflicted by regional-specific serological background and still might be more sensitive to subtle strain-specific changes in the native antigen. The use of ratios associated with serological responses to specific antigens can be used to create more accurate serological profiles that might contribute for identification of infected animals. Disease-specific serological footprints might be exploited to better understand the immune responses of clinically and sub-clinically infected and/or merely exposed animals, enabling early warning system for disease development and more accurate serosurveillance studies. Further studies with different recombinant antigens and experimentally infected animals and cohorts of animals with different clinical status will validate these observations and help to overcome the reported failure of complex antigens mixtures (SPLA) and recombinant proteins (rK39) in establishing meaningful seropositivity levels.

\section{SUPPLEMENTARY MATERIAL}

The supplementary material for this article can be found at https://doi.org/10.1017/S003118 2017000713.

\section{ACKNOWLEDGEMENTS}

Our thanks to Dr Steven Reed, from Infectious Disease Research Institute (Seattle, Washington, USA) for providing the recombinant protein $\mathrm{rK} 39$.

\section{F INANCIAL SUPPORT}

This work was financed by FEDER - Fundo Europeu de Desenvolvimento Regional funds through the COMPETE 2020 - Operacional Programme for Competitiveness and Internationalisation (POCI), Portugal 2020, and by Portuguese funds through FCT - Fundação para a Ciência e a Tecnologia/Ministério da Ciência, Tecnologia e Ensino Superior in the framework of the project 'Institute for Research and Innovation in Health Sciences' (POCI-010145-FEDER-007274) and from the European Community's Seventh Framework Programme under grant agreement No. 603181 [Clinical Studies on a Multivalent Vaccine for Human Visceral Leishmaniasis (MuLeVaClin)].

'This article is a result of the project NORTE-01-0145FEDER-000012, supported by Norte Portugal Regional 
Operational Programme (NORTE 2020), under the PORTUGAL 2020 Partnership Agreement, through the European Regional Development Fund (ERDF)'.

C.L. and N.S. were supported by BD SFRH/BD/ 89183/2012 and European Community's Seventh Framework Programme under grant agreement No. 602773 (Project KINDRED), respectively.

\section{ETHICAL AND REGULATORY GUIDELINES}

This study observed Portuguese legislation for the protection of animals (Law no. 92/1995, from 12 September). According to the European Directive of 24 November 1986, article 2 d, non-experimental, agricultural or clinical veterinary were excluded. The Animal Ethics Committee of the Associate Laboratory IBMC-INEB approved the animal protocol used. Serum samples were collected during vaccination campaigns, and informed consent was obtained from all dog owners before sample collection.

\section{REFERENCES}

Abass, E., Kang, C., Martinkovic, F., Semiao-Santos, S. J., Sundar, S. Walden, P., Piarroux, R., El Harith, A., Lohoff, M. and Steinhoff, U. (2015). Heterogeneity of Leishmania donovani parasites complicates diagnosis of visceral leishmaniasis: comparison of different serological tests in three endemic regions. PLoS ONE 10(3), e0116408. doi: 10.1371/journal.pone. 0116408 .

Arruda, M. M., Figueiredo, F. B., Marcelino, A. P., Barbosa, J. R., Werneck, G. L., Noronha, E. F. and Romero, G. A. (2016). Sensitivity and specificity of parallel or serial serological testing for detection of canine Leishmania infection. Memórias do Instituto Oswaldo Cruz 111(3), 168-173. doi: 10.1590/0074-02760150364.

Bart, A., van Thiel, P.P., de Vries, H. J., Hodiamont, C. J. and Van Gool, T. (2013). Imported leishmaniasis in the Netherlands from 2005 to 2012: epidemiology, diagnostic techniques and sequence-based species typing from 195 patients. Eurosurveillance 18(30), 8 .

Bezuneh, A., Mukhtar, M., Abdoun, A., Teferi, T., Takele, Y., Diro, E., Jemaneh, A., Shiferaw, W., Wondimu, H., Bhatia, A., Howard, R. F., Ghalib, H., Ireton, G. C., Hailu, A. and Reed, S. G. (2014). Comparison of point-of-care tests for the rapid diagnosis of visceral leishmaniasis in East African patients. American Fournal of Tropical Medicine and Hygiene 91(6), 1109-1115. doi: 10.4269/ajtmh.13-0759.

Carvalho, B. M., Rangel, E. F., Ready, P. D. and Vale, M. M. (2015) Ecological Niche Modelling Predicts Southward Expansion of Lutzomyia (Nyssomyia) flaviscutellata (Diptera: Psychodidae: Phlebotominae), Vector of Leishmania (Leishmania) amazonensis in South America, under Climate Change. PLoS ONE 10(11), e0143282. doi: 10.1371/journal. pone. 0143282

Courtenay, O., Carson, C., Calvo-Bado, L., Garcez, L. M. and Quinnell, R. J. (2014). Heterogeneities in Leishmania infantum infection: using skin parasite burdens to identify highly infectious dogs. PLoS Neglected Tropical Diseases 8(1), e2583. doi: 10.1371/journal. pntd.0002583.

Dujardin, J. C., Campino, L., Canavate, C., Dedet, J. P., Gradoni, L., Soteriadou, K., Mazeris, A., Ozbel, Y. and Boelaert, M. (2008). Spread of vector-borne diseases and neglect of leishmaniasis, Europe. Emerging Infectious Diseases 14(7), 1013-1018. doi: 10.3201/ eid1407.071589.

Elshafie, A. I., Mullazehi, M. and Ronnelid, J. (2016). General false positive ELISA reactions in visceral leishmaniasis. Implications for the use of enzyme immunoassay analyses in tropical Africa. Fournal of Immunological Methods 431, 66-71. doi: 10.1016/j.jim.2016.02.007.

Fraga, D. B., Pacheco, L. V., Borja, L. S., Tuy, P. G., Bastos, L. A. Solca Mda, S., Amorim, L. D. and Veras, P. S. (2016). The rapid test based on Leishmania infantum chimeric $\mathrm{rK} 28$ protein improves the diagnosis of canine visceral leishmaniasis by reducing the detection of false-positive dogs. PLoS Neglected Tropical Diseases 10(1), e0004333. doi: 10.1371/journal.pntd.0004333.
Geisweid, K., Weber, K., Sauter-Louis, C. and Hartmann, K. (2013). Evaluation of a conjunctival swab polymerase chain reaction for the detection of Leishmania infantum in dogs in a non-endemic area. Veterinary Fournal 198(1), 187-192. doi: 10.1016/j.tvj1.2013.07.025.

Gomes, Y.M., Paiva Cavalcanti, M., Lira, R. A., Abath, F. G. and Alves, L. C. (2008). Diagnosis of canine visceral leishmaniasis: biotechnological advances. Veterinary Fournal 175(1), 45-52. doi: 10.1016/j. tvj1.2006.10.019.

Maia, C. and Cardoso, L. (2015). Spread of Leishmania infantum in Europe with dog travelling. Veterinary Parasitology 213(1-2), 2-11. doi: 10.1016/j.vetpar.2015.05.003.

Menn, B., Lorentz, S. and Naucke, T. J. (2010). Imported and travelling dogs as carriers of canine vector-borne pathogens in Germany. Parasites $\&$ Vectors 3, 34. doi: 10.1186/1756-3305-3-34

Michalsky, E. M., Rocha, M.F., da Rocha Lima, A.C., FrancaSilva, J. C., Pires, M. Q., Oliveira, F.S., Pacheco, R. S., dos Santos, S. L., Barata, R. A., Romanha, A. J., Fortes-Dias, C. L. and Dias, E.S. (2007). Infectivity of seropositive dogs, showing different clinical forms of leishmaniasis, to Lutzomyia longipalpis phlebotomine sand flies. Veterinary Parasitology 147(1-2), 67-76. doi: 10.1016/j. vetpar.2007.03.004

Millan, J., Ferroglio, E. and Solano-Gallego, L. (2014). Role of wildlife in the epidemiology of Leishmania infantum infection in Europe. Parasitology Research 113(6), 2005-2014. doi: 10.1007/s00436-0143929-2

Miro, G., Checa, R., Montoya, A., Hernandez, L., Dado, D. and Galvez, R. (2012). Current situation of Leishmania infantum infection in shelter dogs in northern Spain. Parasites $\Xi^{\circ}$ Vectors 5, 60. doi: 10.1186/ 1756-3305-5-60.

Molina, R., Amela, C., Nieto, J., San-Andres, M., Gonzalez, F. Castillo, J. A., Lucientes, J. and Alvar, J. (1994). Infectivity of dogs naturally infected with Leishmania infantum to colonized Phlebotomus perniciosus. Transactions of the Royal Society of Tropical Medicine and Hygiene 88(4), 491-493.

Morales-Yuste, M., Morillas-Marquez, F., Diaz-Saez, V., BaronLopez, S., Acedo-Sanchez, C. and Martin-Sanchez, J. (2012). Epidemiological implications of the use of various methods for the diagnosis of canine leishmaniasis in dogs with different characteristics and in differing prevalence scenarios. Parasitology Research 111(1), 155-164. doi: 10.1007/s00436-011-2812-7.

Moreno, J. and Alvar, J. (2002). Canine leishmaniasis: epidemiological risk and the experimental model. Trends in Parasitology 18(9), 399-405. doi: S1471492202023474 [pii].

Mukhtar, M., Abdoun, A., Ahmed, A.E., Ghalib, H., Reed, S. G., Boelaert, M., Menten, J., Khair, M. M. and Howard, R. F. (2015). Diagnostic accuracy of rK28-based immunochromatographic rapid diagnostic tests for visceral leishmaniasis: a prospective clinical cohort study in Sudan. Transactions of the Royal Society of Tropical Medicine and Hygiene 109(9), 594-600. doi: 10.1093/trstmh/trv060.

Naucke, T. J., Menn, B., Massberg, D. and Lorentz, S. (2008) Sandflies and leishmaniasis in Germany. Parasitology Research 103 (Suppl. 1), S65-68. doi: 10.1007/s00436-008-1052-y.

Noli, C. and Saridomichelakis, M. N. (2014). An update on the diagnosis and treatment of canine leishmaniosis caused by Leishmania infantum (syn. L. chagasi). Veterinary Fournal 202(3), 425-435. doi: 10.1016/j. tvj1.2014.09.002.

Palatnik-de-Sousa, C. B. (2012). Vaccines for canine leishmaniasis. Frontiers in Immunology 3, 69. doi: 10.3389/fimmu.2012.00069.

Paltrinieri, S., Solano-Gallego, L., Fondati, A., Lubas, G. Gradoni, L., Castagnaro, M., Crotti, A., Maroli, M., Oliva, G., Roura, X., Zatelli, A., Zini, E. and Canine Leishmaniasis Working Group, I. S. o. V. o. C. A. (2010). Guidelines for diagnosis and clinical classification of leishmaniasis in dogs. Fournal of American Veterinary Medical Association 236(11), 1184-1191. doi: 10.2460/javma.236.11.1184.

Parikh, R., Mathai, A., Parikh, S., Chandra Sekhar, G. and Thomas, R. (2008). Understanding and using sensitivity, specificity and predictive values. Indian fournal of Ophthalmology 56(1), 45-50.

Pattabhi, S., Whittle, J., Mohamath, R., El-Safi, S., Moulton, G. G., Guderian, J. A., Colombara, D., Abdoon, A. O., Mukhtar, M. M., Mondal, D., Esfandiari, J., Kumar, S., Chun, P., Reed, S. G. and Bhatia, A. (2010). Design, development and evaluation of rK28-based point-of-care tests for improving rapid diagnosis of visceral leishmaniasis. PLoS Neglected Tropical Diseases 4(9), e822. doi: 10.1371/journal. pntd.0000822.

Peixoto, H. M., de Oliveira, M. R. and Romero, G. A. (2015). Serological diagnosis of canine visceral leishmaniasis in Brazil: systematic review and meta-analysis. Tropical Medicine \& International Health 20 (3), 334-352. doi: 10.1111/tmi.12429. 
Porrozzi, R., Santos da Costa, M. V., Teva, A., Falqueto, A., Ferreira, A. L., dos Santos, C. D., Fernandes, A. P., Gazzinelli, R.

T., Campos-Neto, A. and Grimaldi, G., Jr. (2007). Comparative evaluation of enzyme-linked immunosorbent assays based on crude and recombinant leishmanial antigens for serodiagnosis of symptomatic and asymptomatic Leishmania infantum visceral infections in dogs. Clinical and Vaccine Immunology 14(5), 544-548. doi: 10.1128/CVI.00420-06.

Ready, P. D. (2010). Leishmaniasis emergence in Europe. Eurosurveillance 15(10), 11

Saari, S., Rasi, J. and Anttila, M. (2000). Leishmaniosis mimicking oral neoplasm in a dog: an unusual manifestation of an unusual disease in Finland. Acta Veterinaria Scandinavica 41(1), 101-104.

Santarem, N., Silvestre, R., Cardoso, L., Schallig, H., Reed, S. G. and Cordeiro-da-Silva, A. (2010). Application of an improved enzymelinked immunosorbent assay method for serological diagnosis of canine leishmaniasis. Fournal of Clinical Microbiology 48(5), 1866-1874. doi: 10.1128/JCM.02402-09.

Saridomichelakis, M. N. (2009). Advances in the pathogenesis of canine leishmaniosis: epidemiologic and diagnostic implications. Veterinary Dermatology 20(5-6), 471-489. doi: VDE823 [pii], 10.1111/j.13653164.2009.00823.x.

Silva, D. T. d., Starke-Buzetti, W. A., Alves-Martin, M.F., Paixão, M. d. S., Tenório, M. d. S. and Lopes, M. L. M. (2014). Comparative evaluation of several methods for Canine Visceral Leishmaniasis diagnosis. Revista Brasileira de Parasitologia Veterinária 23(2), 179-186. doi: 10.1590/s1984-29612014033.
Silvestre, R., Santarem, N., Teixeira, L., Cunha, J., Schallig, H. and Cordeiro-da-Silva, A. (2009). Evaluation of Leishmania species reactivity in human serologic diagnosis of leishmaniasis. American Fournal of Tropical Medicine and Hygiene 81(2), 202-208. doi: 81/2/202 [pii]

Solano-Gallego, L., Miro, G., Koutinas, A., Cardoso, L., Pennisi, M. G. Ferrer, L., Bourdeau, P., Oliva, G., Baneth, G. and The LeishVet, G. (2011). LeishVet guidelines for the practical management of canine leishmaniosis. Parasites $\&$ Vectors 4, 86. doi: 10.1186/1756-3305-4-86.

Sousa, S., Lopes, A. P., Cardoso, L., Silvestre, R., Schallig, H., Reed, S. G. and Cordeiro da Silva, A. (2011). Seroepidemiological survey of Leishmania infantum infection in dogs from northeastern Portugal. Acta Tropica 120(1-2), 82-87. doi: 10.1016/j.actatropica.2011.06.003.

Teske, E., van Knapen, F., Beijer, E. G. and Slappendel, R. J. (2002)

Risk of infection with Leishmania spp. in the canine population in the Netherlands. Acta Veterinaria Scandinavica 43(4), 195-201.

Wylie, C. E., Carbonell-Antonanzas, M., Aiassa, E., Dhollander, S., Zagmutt, F. J., Brodbelt, D. C. and Solano-Gallego, L. (2014). A systematic review of the efficacy of prophylactic control measures for naturally-occurring canine leishmaniosis, part I: vaccinations. Preventive Veterinary Medicine 117(1), 7-18. doi: 10.1016/j.prevetmed.2014.06.015. Zanette, M. F., Lima, V. M., Laurenti, M. D., Rossi, C. N., Vides, J. P., Vieira, R. F., Biondo, A.W. and Marcondes, M. (2014). Serological cross-reactivity of Trypanosoma cruzi, Ehrlichia canis, Toxoplasma gondii, Neospora caninum and Babesia canis to Leishmania infantum chagasi tests in dogs. Revista da Sociedade Brasileira de Medicina Tropical 47(1), 105107. doi: S0037-86822014000100105 [pii], 10.1590/0037-8682-1723-2013. 